\title{
Different Mortality Effects of Extreme Temperature Stress in Three Large City Clusters of Northern and Southern China
}

\author{
Lingyan Zhang ${ }^{1} \cdot{\text { Zhao } \text { Zhang }^{1} \cdot \text { Chenzhi Wang }}^{1} \cdot$ Maigeng Zhou $^{2} \cdot$ \\ Peng Yin ${ }^{2}$
}

Published online: 6 December 2017

(c) The Author(s) 2017. This article is an open access publication

\begin{abstract}
Extreme temperature events have affected Chinese city residents more frequently and intensively since the early 2000s, but few studies have identified the impacts of extreme temperature on mortality in different city clusters of China. This study first used a distributed lag, nonlinear model to estimate the county/district-specific effects of extreme temperature on nonaccidental and cardiovascular mortality. The authors then applied a multivariate meta-analysis to pool the estimated effects in order to derive regional temperature-mortality relationship in three large city clusters-the Beijing-Tianjin-Hebei (BTH) region, the Yangtze River Delta (YRD), and the Pearl River Delta (PRD), which represent northern and southern regions. With 0-3 days' lag, the strongest heat-related mortality effect was observed in the BTH region (with relative risk (RR) of 1.29 ; $95 \%$ confidence interval (CI): $1.13-1.47)$, followed by the YRD $(\mathrm{RR}=1.25$; $95 \% \mathrm{CI}$ : $1.13-1.35)$ and the PRD (RR $=1.14 ; 95 \%$ CI: 1.01-1.28) areas. With 0-21 days' lag, the cold effect was pronounced in all city clusters, with the highest extreme cold-related mortality risk found in the PRD area $(\mathrm{RR}=2.27 ; 95 \% \mathrm{CI}$ : 1.63-3.16), followed by the YRD area $(\mathrm{RR}=1.85 ; 95 \%$ CI: $1.56-2.20)$ and $\mathrm{BTH}$ region $(\mathrm{RR}=1.33 ; 95 \% \mathrm{CI}$ : 0.96-1.83). People in the southern regions tended to be more vulnerable to cold stress, but the northern population
\end{abstract}

Zhao Zhang

zhangzhao@bnu.edu.cn

$1 \quad$ State Key Laboratory of Earth Surface Processes and Resources Ecology/Academy of Disaster Reduction and Emergency Management, Faculty of Geographical Science, Beijing Normal University, Beijing 100875, China

2 National Center for Chronic and Noncommunicable Disease Control and Prevention, Chinese Center for Disease Control and Prevention, Beijing 100050, China was more sensitive to heat stress. By examining the effects of extreme temperature in city clusters of different regions, our findings underline the role of adaptation towards heat and cold, which has important implications for public health policy making and practice.

Keywords China - City cluster - Extreme temperature stress $\cdot$ Health risk $\cdot$ Mortality risk

\section{Introduction}

According to the Fifth Report of the Intergovernmental Panel on Climate Change (IPCC), evidence of climate system warming is unequivocal, and the global mean surface temperatures in $2081-2100$ will be up to $3.7^{\circ} \mathrm{C}$ higher than those of 1986-2005 under the Representative Concentration Pathway (RCP) 8.5 scenario, with an uncertainty range from 2.6 to $4.8{ }^{\circ} \mathrm{C}$ (IPCC 2014). As global warming continues, there is increasing evidence that extreme climatic events are becoming more frequent, more intense, and longer-lasting (Guo et al. 2013; Gao et al. 2015). Extreme heat events have increased human mortality in North America (IPCC 2014) and Europe (Conti et al. 2005; Michelozzi et al. 2009; Lass et al. 2011; Schuster et al. 2014). Cold weather also contributes to a wider range of impacts on public health, including death from respiratory and cardiovascular diseases (CVD) (Braga et al. 2002; Barnett et al. 2012).

The IPCC report claims that in urban areas climate change is projected to increase risks for people, assets, economies, and ecosystems, including risks from heat stress. As the world is increasingly urbanized, the twentyfirst century agenda would have to focus on greatly reducing vulnerabilities of people and infrastructure in 
urban areas to the impacts of a changing climate (IPCC 2014). Due to their dense population and significant economic status, cities have become hot spots of health risk caused by extreme temperature events (Yang et al. 2012; Chen et al. 2015).

A city cluster is defined as a group of cities that are geographically colocated and linked by interdependencies in improving their competitiveness and economic development. In modern China, the collective economic capacity and interdependency of city clusters have influenced regional and national social and economic development (Shao et al. 2006; Zhang 2009). The role of city clusters can be seen in many aspects: comprising only $25 \%$ of China's total area, the 23 city clusters concentrated $62 \%$ of the Chinese population, $80 \%$ of the national GDP, $70 \%$ of investment in fixed assets, $76 \%$ of total retail sales of social consumer goods, and 85\% of college students in 2012. Overall planning for and development of industries, markets, the environment, infrastructure, and social security have been relatively integrated in each city cluster (Fang et al. 2005; Fang 2014). Particularly in recent years, a large number of government policies have been formulated that target the coordinated and integrated development of urban agglomerations and city clusters due to their spatial proximity, economic connections, and the shared climate, environment, culture, and development potential among cities within these clusters. The increasing impact of climate disasters on China's urban areas, especially in the Yangtze River Delta (YRD), the Pearl River Delta (PRD), and the Beijing-Tianjin-Hebei (BTH) region of eastern China and other densely populated urban areas and industrial clusters (Dong et al. 2010; Du et al. 2013), makes it important to examine regional differences in health impacts in order to facilitate the development of regional risk reduction strategies and policies. In contrast to a focus on individual cities, comparison of city clusters as aggregated units of analysis offers a unique advantage for investigating issues and impacts driven by regional scale events, such as climate extremes.

In response to the increasing number of disasters, in 2016 China's National Development and Reform Commission promulgated the City Climate Action Planning program, which proposes to construct climate-adapted cities according to their climatic and geographical conditions and economic and social development. This document identifies and characterizes related risks in urban areas, particularly public health risks; the health risks from extreme temperature events are considered an especially important task for climate action planning (NDRC 2016). Existing studies have examined temperature-mortality relationships according to different study objectives; some have focused on a single city (Guo et al. 2011; Yang et al. 2012; Chen et al. 2015), and others have concentrated on special climatic or geographic zones (Curriero et al. 2002; Li et al. 2014; Gao et al. 2015; Ma, Wang et al.). But few studies have examined the issue in city clusters. Many studies have used a traditional linear threshold modeling strategy, which only provides a partial picture of possibly complex dependencies and cannot effectively capture the relationship between temperature and mortality (Gasparrini et al. 2012; Ma, Wang et al.). Therefore, it is necessary to use advanced statistical modeling techniques to improve our understanding of the association between mortality risk and temperature in different city clusters.

In this study, we examined the effects of extremely high and low temperatures on daily mortality in three city clusters using a two-stage analysis approach. The BeijingTianjin-Hebei region, the Yangtze River Delta, and the Pearl River Delta are the largest and most developed city clusters in eastern China. Given their spatial locations, climatic conditions, and significance with regard to population and economy, these city clusters were selected for our study. Because of the important role city clusters play in China, and the differences in impact of temperature extremes on the city clusters of different regions, our findings may provide useful information for policymakers to develop effective regional-specific responses to climate extremes.

\section{Research Methods}

This study takes the BTH, YRD, and PRD regions as focus areas, and uses daily meteorological and mortality data of county-level administrative units as well as statistical analysis tools. The research employs these data and tools to examine empirical relationships between extreme temperature and mortality, which is used to infer health risks.

\subsection{Data Collection}

The geographic coverage of BTH, YRD, and PRD regions is defined in Ni (2017); the Development Planning of the Yangtze River Delta Urban Agglomeration issued by the National Development and Reform Commission and the Ministry of Housing and Urban-Rural Development in $2016^{1}$; and the New Type of Urbanization of Guangdong Province issued by Guangdong Provincial Department of Housing and Urban-Rural Development and the Guangdong Provincial Development and Reform Commission in 2017. ${ }^{2}$ Moreover, the social economic data in 2014 for the

\footnotetext{
$\overline{1}$ http://www.ndrc.gov.cn/zcfb/zcfbghwb/201606/W0201607155456 38297734.pdf (in Chinese).

2 http://zwgk.gd.gov.cn/006939799/201708/t20170823_719625.html (in Chinese).
} 
Fig. 1 Locations of the study's three city clusters and the 25 county-level administrative units in eastern China

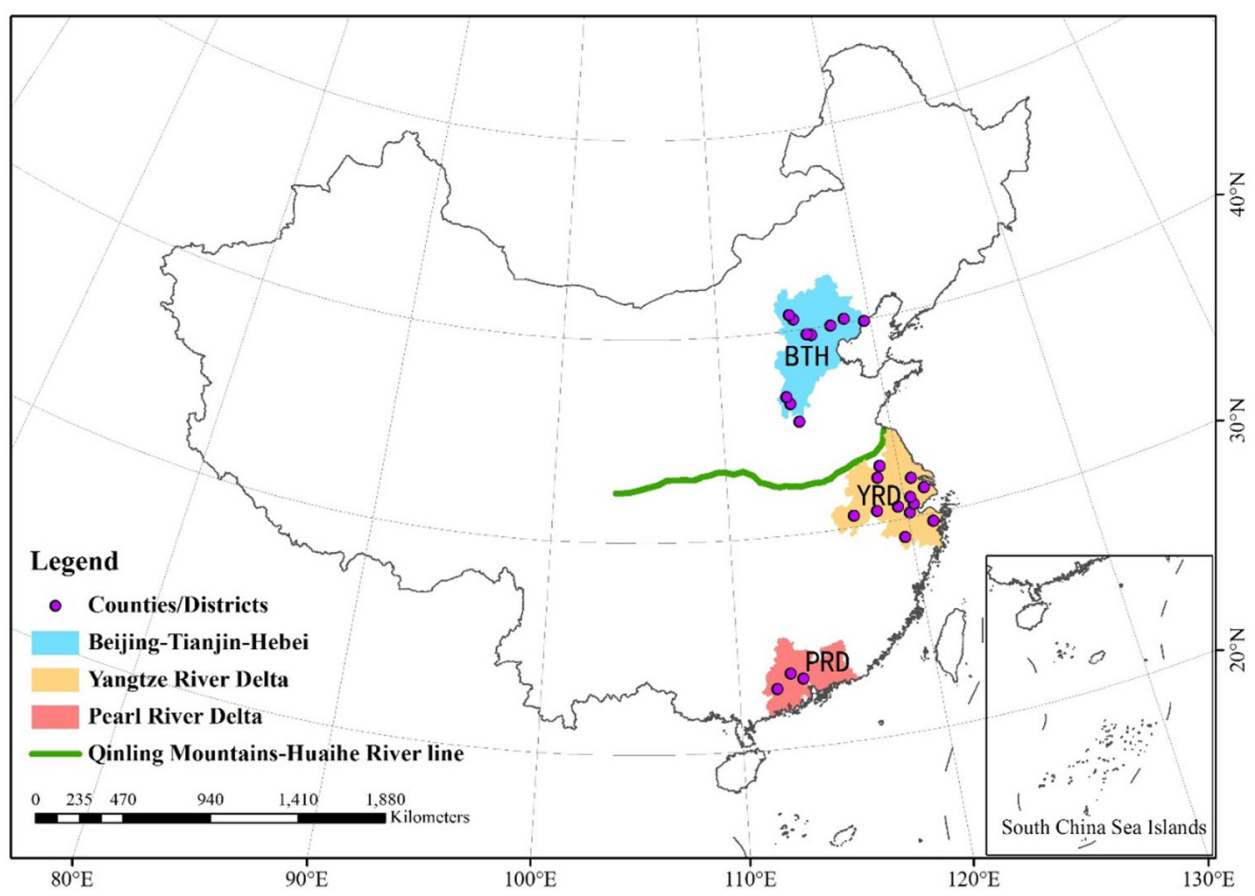

covered cities of the BTH, YRD, and PRD regions were obtained from the China City Statistical Yearbook 2015 (National Bureau of Statistics of China 2015) and included area, population size, and gross domestic product (GDP). Within the three city clusters, a total of 25 county-level cities, counties, and urban districts, where daily mortality data for the study period could be accessed, were selected to represent their respective city cluster and their mortality data were used for the analysis. The BTH, YRD, and PRD regions are characterized by rapid economic development and high levels of urbanization, and represent different climatic zones and geographic features, (Fig. 1). The Qinling Mountains-Huaihe River line is generally accepted as the geographical dividing line between northern and southern China. The BTH region is located in northern China and has a temperate monsoon climate and a cold winter. The YRD is located in eastern China and has a subtropical monsoon climate and four distinct seasons. The PRD is located in southern China and has a subtropical monsoon climate, a warm winter, and a hot summer (Huang et al. 2012; Yang et al. 2016).

The daily mortality data of five county-level cities, eight counties, and twelve urban districts from 1 January 2007 to 31 December 2012 were obtained from the Chinese Center for Disease Control and Prevention. ${ }^{3}$ Cardiovascular mortality (coded as I00-I99 in the 10th International Classification of Diseases) and nonaccidental mortality (defined as deaths not resulting from external causes and coded as A00-R99 by the World Health Organization in (2011) were

\footnotetext{
${ }^{3}$ http://www.chinacdc.cn/ (in Chinese).
}

extracted to create the daily mortality dataset for this study. Data on more counties would improve the estimation accuracy, but daily cardiovascular and nonaccidental mortality data for all other county-level administrative units of the study area were inaccessible to the authors, and (as later statistical tests would prove) 25 county-level units is a sufficiently large sample for our analysis. Therefore, the mortality data of the 25 cities, counties, and districts were used to represent the data of urbanized areas in the three city clusters.

Following the procedure of previous studies (Ma, Wang et al.), a thin-plate spline method was applied by Yuan et al. (2014) to generate daily gridded meteorological variables based on daily meteorological data from 600 national climate stations in the publicly accessible China National Weather Data Sharing System. ${ }^{4}$ The newly generated weather data, which include mean temperature and relative humidity $(\mathrm{RH})$, have superior accuracy, are available at $0.25^{\circ} \times 0.25^{\circ}$ grid divisions, and thus were used in our study. A county/district's daily weather data are derived from average values of all grids covered by the county or district rather than employ a single value from within or around the county or district, as some previous studies have done (Chen et al. 2015; Yang et al. 2016). The averaging method employed in this study is more reasonable, because the derived climate measurements better characterize the spatial variations within a county than those employing station measurements.

\footnotetext{
${ }^{4}$ http://data.cma.cn/site/index.html (in Chinese).
} 


\subsection{Statistical Analysis}

We used a two-stage approach to analyze data in this study. First, a quasi-Poisson regression model, combined with the distributed lag nonlinear model (DLNM), was used to estimate the relationship between temperature and mortality at a county scale. This analysis employed a crossbasis function that describes a bidimensional association between temperature and number of lag days (Gasparrini et al. 2010). To control for potential confounders, the RH, long-term and seasonal trends, day of the week (DOW), and holidays were introduced into the model simultaneously. The temperature-mortality relationship was described using the following model form:

$$
\begin{aligned}
\log E\left(Y_{t}\right)= & \alpha+\operatorname{cb}\left(T_{\text {mean }}, \operatorname{lag}\right)+\mathrm{ns}\left(\mathrm{Rh}_{t}, d f=3\right) \\
& +\mathrm{ns}\left(\text { Time }_{t}, d f\right. \\
= & 9 * 6)+ \text { Dow }_{t}+\text { Holiday }_{t}
\end{aligned}
$$

where $Y_{t}$ refers to the number of deaths on day $t ; \alpha$ is the intercept; $d f$ means degrees of freedom; the degrees of freedom in the model were all chosen by the Akaike information criterion for quasi-Poisson (Q-AIC) (Akaike 1973); cb means cross-basis function, defined by a natural cubic spline with 4 degrees of freedom (df) for the lag space and a quadratic B-spline with $5 \mathrm{df}$ for the temperature space, centered at minimum-mortality temperature (MMT) that corresponds to the lowest mortality risk; $T_{\text {mean }}$ refers to the mean temperature on day $t$; $n s$ means the natural spline function; $\mathrm{Rh}_{t}$ refers to the $\mathrm{RH}$ on day $t$, with $3 \mathrm{df}$; Time $_{t}$ refers to the time variable to adjust for time trends, with $9 \mathrm{df}$ per year; Dow $_{t}$ is a categorical variable for DOW, and Holiday ${ }_{t}$ is the binary variable indicating public holidays.

For each county or district, we fitted a separate DLNM for CVD and nonaccidental death (total mortality minus that from external causes). Then we reduced the first stage's fit of the bidimensional DLNM to the overall cumulative exposure-response relationship, which is associated to the whole exposure history within the lag period (Gasparrini 2011). The relative risk (RR) of mortality at each temperature was calculated by dividing the response value $\left(\log E\left(Y_{t}\right)\right)$ at that temperature by that at the MMT.

In the second stage, a multivariate meta-analysis was applied to pool the county/district-specific estimates to derive the cluster-level and overall nonlinear temperaturemortality relationship, as has been done in previous studies (Gasparrini and Armstrong 2013; Ma, Wang et al.). Relative risk of mortality at the 1st and 99th percentile of temperature was regarded as the extreme cold- and heatrelated mortality risk of each city cluster, respectively. Finally, the maximum extreme temperature-related mortality risk among all lag periods was used to indicate cold and heat effects-the significant mortality increases associated with cold stress and heat stress-respectively.

\subsection{Sensitivity Analysis}

During DLNM analysis, the temperature-mortality association is very sensitive to lag time. We set the lag time as $0-3,0-5,0-7,0-10,0-15,0-21,0-28$, and $0-30$ days to check the robustness of our estimates, then compared the effect of extreme temperature within each grouping of lag days, and selected the reasonable lag days to quantify the cold/heat effect. The concentration of fine particulates in parts per million (PM2.5) and air pollution index (API) were included in the analysis of Beijing and the relative results of air pollution effect are discussed.

The R software package version 3.2.5 (RDCT 2016) was used to perform these analyses. The temperature-mortality associations were estimated using the package dlnm (version 2.3.2), and multivariate meta-analysis was performed using the package mvmeta (version 0.4.7).

\section{Results}

Temperature-mortality relationships of the county/district units were analyzed and this section presents the results at the aggregated city cluster level for these relationships to estimate relative risk. This section also presents the effect of lag days on the estimation results.

\subsection{Statistical Description of the City Clusters}

We summarize the information on the number and name of the county-level cities, counties, and districts, daily average mortality counts for each county/district, meteorological condition, and socioeconomic condition of each city cluster in Table 1. Overall, 477,240 deaths from 2007 to 2012 were recorded in the Chinese Center for Disease Control and Prevention dataset, with the PRD and BTH having the most daily average nonaccidental deaths and highest daily average cardiovascular mortality counts, respectively. Mean temperature and $\mathrm{RH}$ are annual averages from 2007 to 2012, both of them increased from north to south. Socioeconomic conditions in different city clusters were summarized by the area, population, and GDP in 2014. While the three city clusters occupy only $6 \%$ of China's territory and encompass only about $25 \%$ of the country's total population, they accounted for nearly $40 \%$ of the GDP in 2014 (National Bureau of Statistics of China 2015). 
Table 1 Summary mortality, weather statistics, area, population, and economic data in three city clusters in eastern China

\begin{tabular}{|c|c|c|c|}
\hline City cluster & Beijing-Tianjin-Hebei & Yangtze River Delta & Pearl River Delta \\
\hline $\begin{array}{l}\text { Number and } \\
\text { names of } \\
\text { cities/ } \\
\text { districts }\end{array}$ & $\begin{array}{l}10 \\
\text { (Hua County, Anyang; Ci County, Handan; } \\
\text { Wuan City, Handan; Tongzhou District, } \\
\text { Beijing; Dongchen District, Beijing; Ji } \\
\text { County, Tianjin; Haigang District, } \\
\text { Qinhuangdao; Xuanhua County, } \\
\text { Zhangjiakou; Qiaodong District, } \\
\text { Zhangjiakou; Qianxi County, Tangshan) }\end{array}$ & $\begin{array}{l}12 \\
\text { (Wucheng District, Jinhua; Fenhua City, } \\
\text { Ningbo; Daguan District, Anqing; Xiacheng } \\
\text { District, Hangzhou; Jing County, Xuancheng; } \\
\text { Anji County, Huzhou; Tongxiang County, } \\
\text { Jiaxing; Wuzhong District, Suzhou; } \\
\text { Zhangjiagang City, Suzhou; Tianchang City, } \\
\text { Chuzhou; Songjiang District, Shanghai; } \\
\text { Pukou District, Nanjing) }\end{array}$ & $\begin{array}{l}3 \\
\text { (Yuncheng District, } \\
\text { Yunfu; Yuexiu District, } \\
\text { Guangzhou; Sihui City, } \\
\text { Zhaoqing) }\end{array}$ \\
\hline \multicolumn{4}{|c|}{ Daily average mortality counts per county/district } \\
\hline $\begin{array}{l}\text { Nonaccidental } \\
\text { mortality }\end{array}$ & $8(2-24)$ & $9(3-16)$ & $10(4-19)$ \\
\hline $\begin{array}{l}\text { Cardiovascular } \\
\text { mortality }\end{array}$ & $5(1-14)$ & $4(1-7)$ & $4(2-7)$ \\
\hline \multicolumn{4}{|c|}{ Meteorological condition } \\
\hline $\begin{array}{l}\text { Relative } \\
\quad \text { humidity }(\%)\end{array}$ & 57.3 & 72.9 & 74.8 \\
\hline \multicolumn{4}{|l|}{ Temperature $\left({ }^{\circ} \mathrm{C}\right)$} \\
\hline & $11.2(-14.2-30.1)$ & $17.2(-2.6-32.7)$ & $22.1(4.0-31.5)$ \\
\hline SD & 11.6 & 9.1 & 6.6 \\
\hline \multicolumn{4}{|c|}{ Socioeconomic conditions } \\
\hline Megacities & Beijing, Tianjin, Tangshan & Shanghai, Nanjing, Hangzhou & Guangzhou, Shenzhen \\
\hline $\begin{array}{l}\text { Area } \\
\qquad\left(1000 \mathrm{~km}^{2}\right)\end{array}$ & 222.3 & 211.7 & 181.0 \\
\hline $\begin{array}{l}\text { Population } \\
\text { (million) }\end{array}$ & 115.6 & 144.0 & 72.5 \\
\hline $\begin{array}{l}\text { Percentage in } \\
\text { national } \\
\text { population }\end{array}$ & $8.5 \%$ & $10.5 \%$ & $5.3 \%$ \\
\hline $\begin{array}{l}\text { GDP (CNY } \\
\text { billion) }\end{array}$ & 6835.7 & $12,670.0$ & 6216.6 \\
\hline $\begin{array}{l}\text { GDP per capita } \\
\text { (CNY) }\end{array}$ & $59,132.3$ & $87,986.1$ & $85,746.2$ \\
\hline $\begin{array}{l}\text { Percentage in } \\
\text { national } \\
\text { GDP }\end{array}$ & $10.7 \%$ & $18.5 \%$ & $9.8 \%$ \\
\hline
\end{tabular}

Numbers in parentheses indicate range of county unit's mortality and temperature

Data source Daily mortality data were recorded by the Chinese Center for Disease Control and Prevention (http://www.chinacdc.cn/), daily meteorological data were obtained from the China National Weather Data Sharing System (http://data.cma.cn/site/index.html), and the socioeconomic data were extracted from the China City Statistical Yearbook (National Bureau of Statistics of China 2015)

\subsection{Sensitivity of Lag Days}

Figure 2 shows the extreme cold/heat-related mortality risk (relative risk) with different lag days in the three city clusters and the overall study area. The confidence intervals of extreme cold/heat-related mortality risk become wider with the increased maximum lag time. The highest extreme cold-related mortality risk was found at lag 0-21 days in most regions except in the PRD. Although the extreme cold-related mortality risks at lag 0-28 and 0-30 days are higher than that at lag 0-21 days in the PRD, its confidence intervals also changed hugely especially for CVD, suggesting an aggravated uncertainty. The extreme heat-related mortality risk decreased slightly or has little change with maximum lag time increasing. In general, the optimal maximum lag time should be placed at the day where the shape of the temperature-mortality curve will not change significantly even when longer lag time is applied. According to the sensitivity analysis and previous studies (Guo et al. 2011; Wu et al. 2013; Ma, Zeng et al. ; Wang et al. 2017), 0-3 and 0-21 days were finally selected as the 


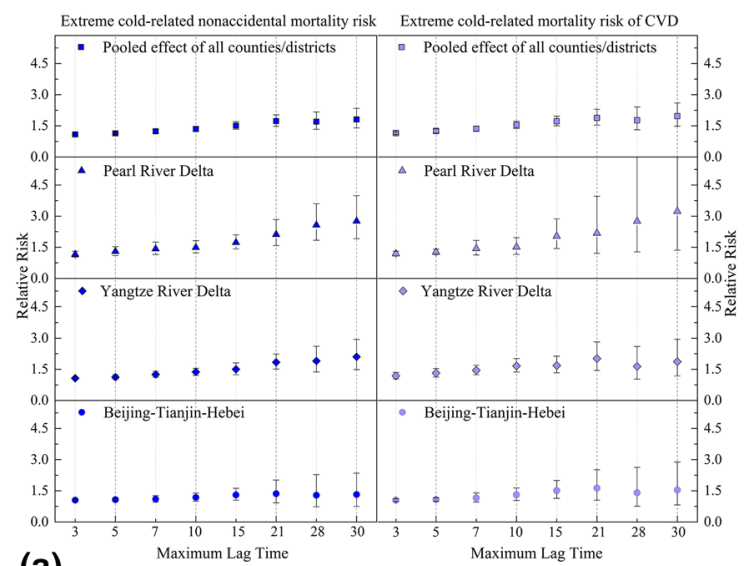

(a)

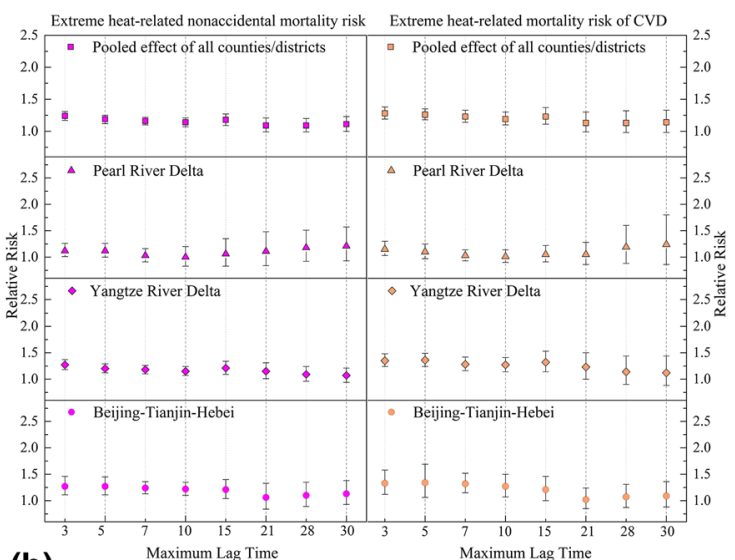

(b)

Fig. 2 Sensitivity analysis of extreme cold/heat-related mortality risks with confidence intervals of different maximum lag time in three city clusters in eastern China. a Lag effect of extreme cold-related mortality risk. b Lag effect of extreme heat-related mortality risk

maximum lag times for quantifying the cold/heat effect, respectively, in this study.

\subsection{Pooled Temperature-Mortality Relationships of All Counties/Districts}

In order to first depict an overall picture of the temperature-mortality relationships for all city clusters, Fig. 3 shows the relative risks (RRs) of pooled averages of all counties and districts from multivariate meta-analysis with the $0-3$ and $0-21$ days' lags. Given the wide temperature range of the city clusters across eastern China, temperature percentile instead of actual temperature is used to plot the curves. The pooled relationships between temperature and mortality are U- and L-shaped with the 0-3 and 0-21 days lags, respectively. The heat effect was obvious with the 0-3 days' lag, indicating that high temperature affects mortality relatively rapidly. However, high RRs were observed for mortality at low temperature with the 0-21 days' lag, meaning that the cold effect took place relatively slowly and could last 3 weeks. For the pooled nonaccidental and CVD curves in Fig. 3, the pooled RRs for extremely high temperature were 1.24 (95\% CI: 1.17-1.31) and 1.28 (95\% CI: 1.19-1.38), respectively. The pooled RRs for extremely low temperature were 1.73 (95\% CI: $1.48-2.03$ ) and 1.94 (95\% CI: 1.61-2.33), respectively.

\subsection{Pooled Temperature-Mortality Relationships of City Clusters}

Figure 4 illustrates the effects of temperature on mortality, examined with both the 0-3 and the 0-21 days' lags in each city cluster. With the 0-3 days' lag, the curves are basically $\mathrm{U}$ or V shapes in the city clusters, with the BTH region and the YRD showing a relatively higher extreme heat-related mortality risk, while the PRD region reacts quickly to cold stress. With the 0-21 days' lag, the curve shapes are "W" in the BTH region and "L" in the YRD and PRD, with a consistently higher extreme cold-related mortality risk for all city clusters than that of heat. Among the city clusters, the cold effects are stronger in the two southern city clusters (YRD and PRD).

The MMTs, which were lower in northern China, are also displayed in Fig. 4. Specifically, for the 0-3 lag days, MMT for nonaccidental mortality was $1{ }^{\circ} \mathrm{C}$ in the BTH region, $12.4{ }^{\circ} \mathrm{C}$ in the YRD, and $18{ }^{\circ} \mathrm{C}$ in the PRD. Minimum mortality temperatures with the $0-3$ days' lag were consistently lower than those with the 0-21 days' lag; and the MMTs were generally close to the 25 th and 75 th percentiles of local ambient temperature with the lag days of $0-3$ and $0-21$, respectively.

\subsection{Cold and Heat Effects}

According to the sensitivity test of different lag days, in this study we quantified the "heat effect" as the extreme heat-related mortality risk with $0-3$ lag days; and we used the models with lags of 0-21 days to calculate the extreme cold-related mortality risk as the "cold effect." The results for each city cluster are displayed in Fig. 5 and Table 2.

The results indicate a higher risk of CVD than nonaccidental mortality under extreme temperature events in all city clusters. From northern China to southern China, the cold effects became stronger-the relative risk (and 95\% confidence interval) was $1.33 \quad(0.96-1.83), 1.85$ (1.56-2.20), and 2.27 (1.63-3.16) for BTH, YRD, and PRD, respectively, and the heat effects became weakerthe relative risk was 1.29 (1.13-1.47), $1.25(1.13-1.35)$, and 1.14 (1.01-1.28) for BTH, YRD, and PRD, respectively. Such results imply that the northern population is 

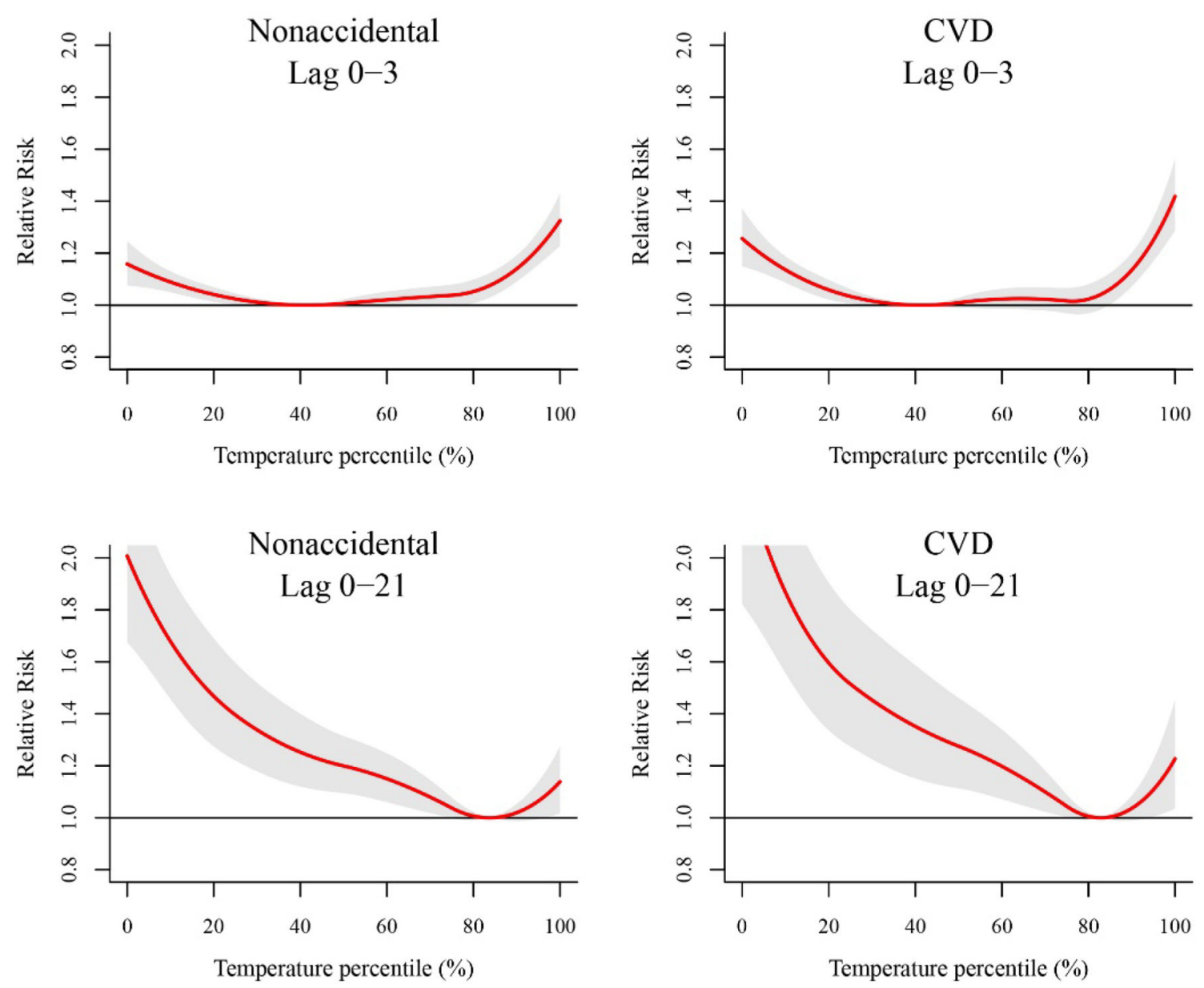

Fig. 3 Pooled effects of temperature on nonaccidental and cardiovascular disease (CVD) mortality with 0-3 and 0-21 lag days from 25 counties/districts in BTH, YRD, and PRD, with reference to mortality

more vulnerable to heat stress and southern city residents are more sensitive to extreme cold events.

\section{Discussion}

In this section alternative approaches for estimating cold/ heat effects are first examined. Then outstanding issues, such as regional differences in people's vulnerability to cold and heat stress and reasons for these variations are further elaborated. The need for a balanced focus on both cold and heat risks, as well as the impact of air pollution on mortality risk, is also discussed.

\subsection{Identifying Cold/Heat Effect Using Alternative Approaches}

Many previous studies have reported that heat effects appeared immediately and lasted for about three days (Anderson and Bell 2009; Guo et al. 2011, 2014; Gao et al.

risk at the minimum-mortality temperature (MMT) of the temperature distribution; the pooled results are shown as smooth red lines and the gray areas are $95 \%$ confidential intervals

2015), but cold effects were delayed and lasted for about 3 weeks or even longer (Analitis et al. 2008; Gasparrini and Armstrong 2013; Ma, Wang et al.). Therefore, it is very important to identify a reasonable lag time that captures accurately the magnitude of the cold/heat effect.

Many other studies have chosen the same lag time, such as 0-21 days (Guo et al. 2014; Ma, Wang et al. 2015a; Yang et al. 2016), to calculate extreme heat/cold-related mortality risk. This practice would appear to underestimate the magnitude of the heat effect (acute and last only for a few days) and may not adequately explore the impact of heat stress. Apart from the lag time(s) on which a study is based, quantitative analysis methods can also affect the calculated cold/heat effects. Guo et al. (2011) and Hajat et al. (2007) regarded the relative risk increase in mortality for a $1{ }^{\circ} \mathrm{C}$ temperature decrease below the cold threshold and that for a $1{ }^{\circ} \mathrm{C}$ of temperature increase above the hot threshold as the cold and heat effects, respectively. Therefore, we calculated the cold/heat effects by the same methods suggested by Ma, Wang et al. and Guo et al. 
Fig. 4 Pooled effects of temperature on nonaccidental and cardiovascular disease (CVD) mortality with $0-3$ and 0-21 lag days in each city cluster, with reference to mortality risk at the minimummortality temperature (MMT) of the temperature distribution. The pooled results are shown as smooth red lines, and the gray areas are $95 \%$ confidential intervals. The light blue points on the curves are the MMT points (the specific values are shown above the points), and the three black points from left to right on the curves represent the 25th, 50th, and 75th percentile of the temperature distribution, respectively. a 0-3 days' lag. b 0-21 days' lag
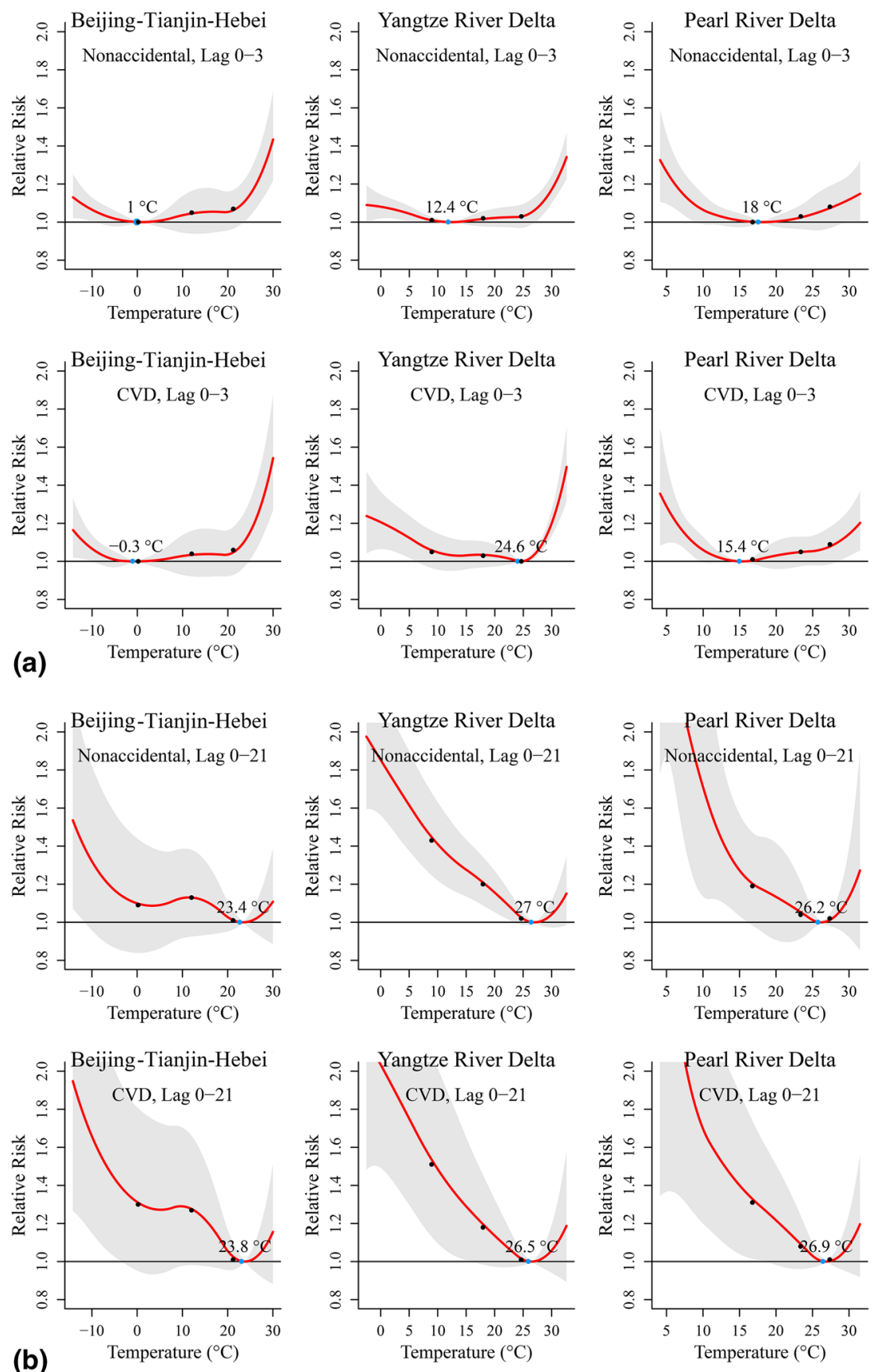

(2011) and compared the results of different approaches. The calculated results for the three city clusters are summarized in Table 3.

The cold and heat effects for nonaccidental mortality in all counties/districts calculated by the approach that Guo et al. (2011) suggested are 1.07 (95\% CI: 1.02-1.11) and 1.08 (95\% CI: $1.03-1.13$ ), which are both very close to 1 . It would be hard to distinguish the impact degree of cold and heat stress on local residents. Furthermore, the relative risks are all very small and close to each other across the three city clusters, which increases the difficulty of finding out the regional characteristics of cold/heat effect. The overall heat effect was 1.08 (95\% CI: 1.00-1.16) in all counties/districts of the three city clusters calculated by the approach used by Ma, Wang et al., which is very weak and lower than that in our study (1.24 (95\% CI: 1.17-1.31)). The underestimated heat effects ( $\mathrm{Ma}$, Wang et al.) make it impossible to compare regional differences. Therefore, the approach applied in this study is reasonable, which can better capture the magnitude and regional characteristic of cold/heat effect. 


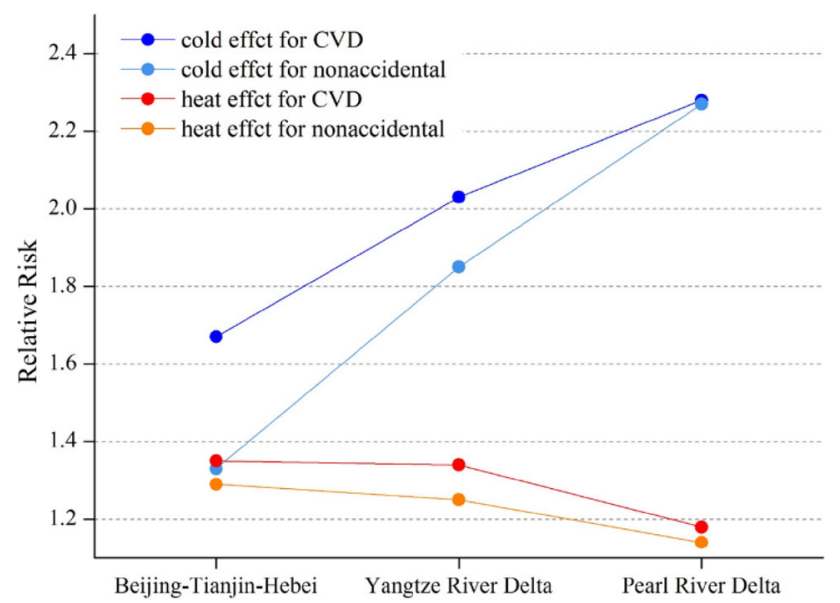

Fig. 5 Cold/heat effects in the three city clusters of eastern China

\subsection{Regional Differences in People's Vulnerability to Cold and Heat Stresses}

The population of southern China tends to be more vulnerable to low temperatures, and residents in the north are more sensitive to heat (Fig. 5). This regional difference in vulnerability is consistent with other studies conducted in Europe, the United States, and China (Curriero et al. 2002; Analitis et al. 2008; Guo et al. 2013; Ma, Wang et al.). Among the three city clusters, the BTH region and the PRD had a quicker response to heat stress and cold stress, respectively. The different responses to temperature extremes between residents of northern and southern China are both a result of and have further contributed to their respective ability to adapt to their local climates. Such adaptation may occur either by acclimatization, behavioral change, technology uptake, or other adaptive mechanisms (Keatinge et al. 2000; Medina-Ramón and Schwartz 2007). For example, central heating, an important adaptive measure against cold, is almost universal in northern China, which may explain why northern residents seemed more acclimatized to cold than the southern population. Central heating is rare in southeastern and southern China, however, and the heating function of air conditioning units may not work well under extreme cold events. Thus, the vulnerability of southern China residents to low temperatures could be explained by their limited adaptive measures to cold. Air conditioning is less prevalent in the BTH region than the YRD and the PRD, which provides a partial explanation of northern China residents' susceptibility to extremely high temperatures.

\subsection{The Need for a Balanced Focus on Both Cold and Heat Risks}

In our study, CVD showed a higher susceptibility to extreme cold in all city clusters (Table 2). Associations of higher CVD risk with cold have also been commonly found in previous studies (Medina-Ramón et al. 2006; Son et al.

Table 2 Pooled cold and heat effects of temperature on nonaccidental mortality and CVD in the three city clusters of eastern China

\begin{tabular}{|c|c|c|c|c|}
\hline & \multicolumn{4}{|c|}{ Relative risk (RR) $(95 \% \mathrm{CI})$} \\
\hline & \multicolumn{2}{|l|}{ Cold effect ${ }^{\mathrm{a}}$} & \multicolumn{2}{|l|}{ Heat effect ${ }^{b}$} \\
\hline & Nonaccidental & Cardiovascular & Nonaccidental & Cardiovascular \\
\hline Beijing-Tianjin-Hebei & $1.33(0.96,1.83)$ & $1.67(1.16,2.41)$ & $1.29(1.13,1.47)$ & $1.35(1.15,1.58)$ \\
\hline Yangtze River Delta & $1.85(1.56,2.20)$ & $2.03(1.49,2.77)$ & $1.25(1.13,1.35)$ & $1.34(1.22,1.46)$ \\
\hline Pearl River Delta & $2.27(1.63,3.16)$ & $2.28(1.35,3.85)$ & $1.14(1.01,1.28)$ & $1.18(1.05,1.32)$ \\
\hline
\end{tabular}

Numbers in parentheses indicate mortality risk at $95 \%$ confidence interval

${ }^{a}$ Mortality risk at the 1 st percentile of temperature relative to that at the MMT, with the 0-21 days' lag

${ }^{\text {b}}$ Mortality risk at the 99th percentile of temperature relative to that at the MMT, with the 0-3 days' lag

Table 3 The calculated cold/heat effects for nonaccidental mortality of the three city clusters using different methods

\begin{tabular}{llllll}
\hline & Approach & Beijing-Tianjin-Hebei & Yangtze River Delta & Pearl River Delta & Overall \\
\hline Cold effect & Guo et al. (2011) & $1.08(0.98,1.19)$ & $1.04(0.98,1.11)$ & $1.04(0.96,1.14)$ & $1.07(1.02,1.11)$ \\
& Ma, Wang et al. & $1.33(0.96,1.96)$ & $1.85(1.56,2.20)$ & $2.27(1.63,3.16)$ & $1.73(1.50,1.99)$ \\
& Our study & $1.33(0.96,1.96)$ & $1.85(1.56,2.20)$ & $2.27(1.63,3.16)$ & $1.73(1.50,1.99)$ \\
Heat effect & Guo et al. (2011) & $1.13(1.02,1.25)$ & $1.09(1.03,1.16)$ & $1.07(0.97,1.19)$ & $1.08(1.03,1.13)$ \\
& Ma, Wang et al. & $1.05(0.91,1.21)$ & $1.09(0.98,1.22)$ & $1.21(0.88,1.65)$ & $1.08(1.00,1.16)$ \\
& Our study & $1.29(1.13,1.47)$ & $1.25(1.17,1.35)$ & $1.14(1.01,1.28)$ & $1.24(1.17,1.31)$ \\
\hline
\end{tabular}

Numbers in parentheses indicate mortality risk at $95 \%$ confidence interval 
Table 4 Comparison of heat and cold effects with and without including PM2.5 and API in the temperature-mortality model of Beijing

\begin{tabular}{|c|c|c|c|c|}
\hline \multirow[t]{3}{*}{ Air pollution adjustment } & \multicolumn{4}{|c|}{ Relative risk (RR) $(95 \% \mathrm{CI})$} \\
\hline & \multicolumn{2}{|l|}{ Cold effect ${ }^{a}$} & \multicolumn{2}{|l|}{ Heat effect ${ }^{b}$} \\
\hline & Nonaccidental & Cardiovascular & Nonaccidental & Cardiovascular \\
\hline Without PM2.5 and API & $1.77(0.81-3.87)$ & $2.20(0.78-6.19)$ & $1.39(1.18-1.64)$ & $1.45(1.17-1.80)$ \\
\hline With PM2.5 and API & $1.80(0.82-3.95)$ & $2.35(0.83-6.63)$ & $1.40(1.17-1.68)$ & $1.46(1.17-1.83)$ \\
\hline
\end{tabular}

Numbers in parentheses indicate mortality risk at the $95 \%$ confidence interval

${ }^{a}$ Mortality risk at the 1 st percentile of temperature relative to that at the MMT, with the 0-21 days' lag

${ }^{b}$ Mortality risk at the 99th percentile of temperature relative to that at the MMT, with the 0-3 days' lag

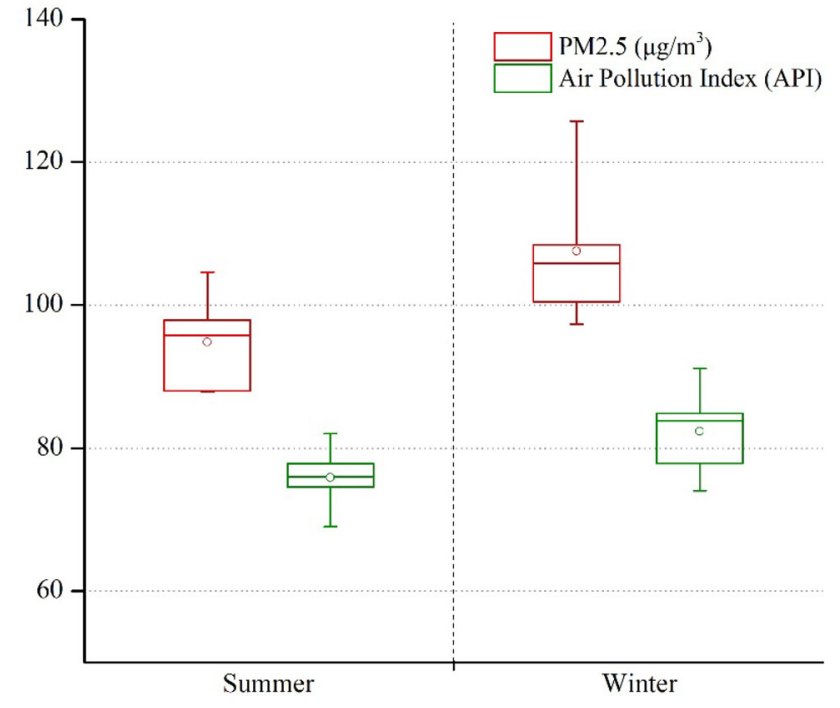

Fig. 6 Average level of PM2.5 and Air Pollution Index (API) in summer (June, July, and August) and winter (December, January, and February) of Beijing from 2007 to 2012. The dashes and open-circles in the boxes are the median and mean values. Boxes and whiskers present 25 to 75 and 10 to 90 percentiles of seasonal pollutants over 2007-2012. Data source Daily PM2.5 levels were recorded by the U.S. embassy in Beijing (https://data.epmap.org/usembassy_air) and API values were reported by the Ministry of Environmental Protection (http://www.zhb.gov.cn/ (in Chinese))

2011). The underlying mechanism for the CVD increase with cold temperatures may be related to the fact that blood pressure is elevated during the winter. Moreover, human plasma cholesterol and plasma fibrinogen levels have been reported to increase upon exposure to cold temperatures, which could lead to thrombosis (Medina-Ramón et al. 2006).

Previous studies have mainly focused on heat waves, with relatively less focus on cold stress. Medina-Ramón and Schwartz (2007) even argued that decreases in coldrelated mortality as a result of decreasing frequency of extreme cold would compensate for increases in heat-related mortality. In our study, the cold effect appeared more obvious than the heat effect in all city clusters (Table 2), and was consistent with the results of Anderson and Bell (2009), Guo et al. (2013), and Ma, Wang et al. . Therefore, considering the high sensitivity of population to cold stress, more attention should be paid to both types of extreme events, rather than only heat waves.

\subsection{Limitations and Implications}

Some of the present study's limitations need to be considered. It was impossible for us to obtain daily air pollution data during the period of more than 10 years across all city clusters because of a strict confidentiality rule on such data in China. Especially, data on ozone, which has potential effects during high temperature days (Chen et al. 2015), are desired but unavailable. As an alternative, we collected data on the concentration of fine particulates (PM2.5) and API in Beijing, and tested the impact of these two indices on mortality by comparing the risks of extreme temperature with versus without air pollutants in Beijing. The daily PM2.5 levels and API were recorded by the U.S. embassy in Beijing ${ }^{5}$ and by the Ministry of Environmental Protection of the People's Republic of China, ${ }^{6}$ respectively. The API that excludes PM2.5 in monitoring was used to report the status of ambient air quality before 2012 in China.

Some previous studies have suggested that temperature effects on health outcomes are generally robust or even independent of air pollution (Anderson and Bell 2009; Yang et al. 2012), but others have found interactive or synergistic effects between temperature and air pollution leading to some sort of hyper-additive mortality effect in Europe (Keatinge et al. 2000; Burkart et al. 2013) and the United States (Ren et al. 2008). Table 4 displays the comparison of extreme temperature effects with and without pollutions in our study, indicating that air pollution could aggravate the extreme cold-related CVD mortality risk (increased from 2.20 to 2.35 when considering the impact of air pollution) but does not have significant

\footnotetext{
5 https://data.epmap.org/usembassy_air.

${ }^{6}$ http://www.zhb.gov.cn/.
} 
impact on the extreme heat-related mortality risk (1.45 vs. 1.46 for relative mortality risk without and with consideration of air pollution). Figure 6 shows the box plot for PM2.5 concentration and API in the summer (June, July, and August) and the winter (December, January, and February) from 2007 to 2012, reflecting that both the level of PM2.5 and API were higher in the winter than the summer. Therefore, the small possible difference between the impacts of air pollution on extreme cold- and heatrelated mortality risks might be attributable to the relatively good air quality in the summer but a higher level of pollution in the winter of Beijing (Table 4 and Fig. 6).

\section{Conclusion}

Based on the study of the three largest Chinese city clusters, we found that residents of southern China tended to be more vulnerable to low temperature stress, but that the northern population was more sensitive to heat stress. Furthermore, the extreme cold-related mortality risk was consistently higher than that of heat. Therefore, it is important to develop different public health policies such as early warning/forecasting system, climate-adapted plan (NDRC 2016), among others, for both extreme cold and heat events, and for different regions according to their specific local climates.

Future research should examine the impact of potential confounding factors. For example, due to the growing concern about air pollution in China, we intend to explore the effect of PM2.5 on human health in further studies. In addition, the temperature-mortality association could be modified by factors such as gender, age, educational level, and the availability of air conditioning and central heating, which were not considered in the present study but can be examined and controlled for in further research.

Acknowledgements This study was supported by the fund for Creative Research Groups of the National Natural Science Foundation of China (No. 41621061) and State Key Laboratory of Earth Surface Processes and Resources Ecology.

Open Access This article is distributed under the terms of the Creative Commons Attribution 4.0 International License (http://crea tivecommons.org/licenses/by/4.0/), which permits unrestricted use, distribution, and reproduction in any medium, provided you give appropriate credit to the original author(s) and the source, provide a link to the Creative Commons license, and indicate if changes were made.

\section{References}

Analitis, A., K.A. Katsouyanni, M. Baccini, B. Forsberg, L. Bisanti, U. Kirchmayer, F. Ballester, E. Cadum, et al. 2008. Effects of cold weather on mortality: Results from 15 European cities within. American Journal of Epidemiology 168(12): 1397-1408.

Akaike, H. 1973. Information theory and the extension of the maximum likelihood principle. In Proceedings of the International Symposium on Information Theory, ed. B.N. Petrov and F. Czaki, 267-281. Akademia Kiadoo, Budapest, Hungary.

Anderson, B.G., and M.L. Bell. 2009. Weather-related mortality: How heat, cold, and heat waves affect mortality in the United States. Epidemiology 20(2): 205-213.

Barnett, A.G., S. Hajat, A. Gasparrini, and J. Rocklöv. 2012. Cold and heat waves in the United States. Environmental Research 112: 218-224.

Braga, A.L.F., Z. Antonella, and S. Joel. 2002. The effect of weather on respiratory and cardiovascular deaths in 12 U.S. cities. Environmental Health Perspectives 110(9): 859-863.

Burkart, K., P. Canário, S. Breitner, A. Schneider, K. Scherber, H. Andrade, M.J. Alcoforado, and W. Endlicher. 2013. Interactive short-term effects of equivalent temperature and air pollution on human mortality in Berlin and Lisbon. Environmental Pollution 183: 54-63.

Chen, K., J. Bi, J. Chen, X. Chen, L. Huang, and L. Zhou. 2015. Influence of heat wave definitions to the added effect of heat waves on daily mortality in Nanjing, China. Science of the Total Environment 506-507: 18-25.

Conti, S., P. Meli, G. Minelli, R. Solimini, V. Toccaceli, M. Vichi, C. Beltrano, and L. Perini. 2005. Epidemiologic study of mortality during the summer 2003 heat wave in Italy. Environmental Research 98(3): 390-399.

Curriero, F.C., K.S. Heiner, J.M. Samet, S.L. Zeger, L. Strug, and J.A. Patz. 2002. Temperature and mortality in 11 cities of the eastern United States. American Journal of Epidemiology 155(1): 80-87.

Dong, S., S. Tao, W. Yang, F. Li, S. Li, Y. Li, and H. Liu. 2010. Impacts of climate change on urban agglomerations in coastal region of China. Advances in Climate Change Research 6(4): 284-289.

Du, Y.D., X.H. Cheng, X.W. Wang, H. Ai, H.L. Duan, J. He, and X.X. Wu. 2013. A review of assessment and adaptation strategy to climate change impacts on the coastal areas in south China. Advances in Climate Change Research 4(4): 201-207.

Fang, C. 2014. Progress and the future direction of research into urban agglomeration in China. Acta Geographica Sinica 69(8): 1130-1144 (in Chinese).

Fang, C., J. Song, Q. Zhang, and M. Li. 2005. The formation, development and spatial heterogeneity patterns for the structures system of urban agglomerations in China. Acta Geographica Sinica 60(5): 827-840 (in Chinese).

Gao, J., Y. Sun, Q. Liu, M. Zhou, Y. Lu, and L. Li. 2015. Impact of extreme high temperature on mortality and regional level definition of heat wave: A multi-city study in China. Science of the Total Environment 505: 535-544.

Gasparrini, A. 2011. Distributed lag linear and non-linear models in R: The package dlnm. Journal of Statistical Software 43(8): $1-20$.

Gasparrini, A., and B. Armstrong. 2013. Reducing and metaanalysing estimates from distributed lag non-linear models. BMC Medical Research Methodology 13(1). https://doi.org/10. 1186/1471-2288-13-1.

Gasparrini, A., B. Armstrong, and M.G. Kenward. 2010. Distributed lag non-linear models. Statistics in Medicine 29(21): 2224-2234.

Gasparrini, A., B. Armstrong, and M.G. Kenward. 2012. Multivariate meta-analysis for non-linear and other multi-parameter associations. Statistics in Medicine 31(29): 3821-3839.

Guo, Y., A.G. Barnett, X. Pan, W. Yu, and S. Tong. 2011. The impact of temperature on mortality in Tianjin, China: A case-crossover design with a distributed lag nonlinear model. Environmental Health Perspectives 119(12): 1719-1725. 
Guo, Y., A. Gasparrini, B. Armstrong, S. Li, B. Tawatsupa, A. Tobias, E. Lavigne, M. Leone, et al. 2014. Global variation in the effects of ambient temperature on mortality: A systematic evaluation. Epidemiology 25(6): 781-789.

Guo, Y., S. Li, Y. Zhang, B. Armstrong, J.J.K. Jaakkola, S. Tong, and X. Pan. 2013. Extremely cold and hot temperatures increase the risk of ischaemic heart disease mortality: Epidemiological evidence from China. Heart 99(3): 195-203.

Hajat, S., R.S. Kovats, and K. Lachowycz. 2007. Heat-related and cold-related deaths in England and Wales: Who is at risk? Occupational and Environmental Medicine 64(2): 93-100.

Huang, R., J. Chen, L. Wang, and Z. Lin. 2012. Characteristics, processes, and causes of the spatio-temporal variabilities of the East Asian monsoon system. Advances in Atmospheric Sciences 29(5): 910-942.

IPCC (Intergovernmental Panel on Climate Change). 2014. Climate change 2014: Synthesis report. Contribution of Working Groups I, II and III to the Fifth assessment report of the Intergovernmental Panel on Climate Change, ed. R.K. Pachauri and L.A. Meyer. Geneva: IPCC.

Keatinge, W.R., G.C. Donaldson, E. Cordioli, M. Martinelli, A.E. Kunst, J.P. Mackenbach, S. Nayha, and I. Vuori. 2000. Heat related mortality in warm and cold regions of Europe: Observational study. BMJ Clinical Research 321(7262): 670-673.

Lass, W., A. Haas, J. Hinkel, and C. Jaeger. 2011. Avoiding the avoidable: Towards a European heat waves risk governance. International Journal of Disaster Risk Science 2(1): 1-14.

Li, Y., Y. Cheng, G. Cui, C. Peng, X. Yan, Y. Wang, Y. Liu, J. Liu, et al. 2014. Association between high temperature and mortality in metropolitan areas of four cities in various climatic zones in China: A time-series study. Environmental Health 13(1): 1-10.

Ma, W., L. Wang, H. Lin, T. Liu, Y. Zhang, S. Rutherford, Y. Luo, W. Zeng, et al. 2015. The temperature-mortality relationship in China: An analysis from 66 Chinese communities. Environmental Research 137: 72-77.

Ma, W., W. Zeng, M. Zhou, L. Wang, S. Rutherford, H. Lin, T. Liu, Y. Zhang, et al. 2015. The short-term effect of heat waves on mortality and its modifiers in China: An analysis from 66 communities. Environment International 75: 103-109.

Medina-Ramón, M., and J. Schwartz. 2007. Temperature, temperature extremes, and mortality: A study of acclimatisation and effect modification in 50 US cities. Occupational and Environmental Medicine 64(12): 827-833.

Medina-Ramón, M., A. Zanobetti, D.P. Cavanagh, and J. Schwartz. 2006. Extreme temperatures and mortality: Assessing effect modification by personal characteristics and specific cause of death in a multi-city case-only analysis. Environmental Health Perspectives 114(9): 1331-1336.

Michelozzi, P., G. Accetta, S.M. De, D. D’Ippoliti, C. Marino, M. Baccini, A. Biggeri, H.R. Anderson, et al. 2009. High temperature and hospitalizations for cardiovascular and respiratory causes in 12 European cities. American Journal of Respiratory and Critical Care Medicine 179(5): 383-389.

National Bureau of Statistics of China. 2015. China city statistical yearbook 2015. Pages 13-19, 83-90, 98-104 (Table 2-1, Table 2-11 and Table 2-13). China Statistics Press, Beijing.
NDRC (National Development and Reform Commission). 2016. City climate action planning. Beijing: NDRC. http://www.ndrc.gov. cn/gzdt/201608/W020160804337181231042.pdf. Accessed 31 Oct 2017.

$\mathrm{Ni}$, W. 2017. New type of urbanization and its coordinated development measuring_-Taking Beijing-Tianjin-Hebei urban agglomeration as an example. Jiangsu Agricultural Sciences 45(8): 317-323 (in Chinese).

RDCT (R Development Core Team). 2016. R: A language and environment for statistical computing. Vienna: R Foundation for Statistical Computing.

Ren, C., G.M. Williams, K. Mengersen, L. Morawska, and S. Tong. 2008. Does temperature modify short-term effects of ozone on total mortality in 60 large eastern US communities? An assessment using the NMMAPS data. Environment International 34(4): 451-458.

Schuster, C., K. Burkart, and T. Lakes. 2014. Heat mortality in Berlin-Spatial variability at the neighborhood scale. Urban Climate 10(part 1): 134-147.

Shao, M., X. Tang, Y. Zhang, and W. Li. 2006. City clusters in China: Air and surface water pollution. Frontiers in Ecology and the Environment 4(7): 353-361.

Son, J.Y., J.T. Lee, G.B. Anderson, and M.L. Bell. 2011. Vulnerability to temperature-related mortality in Seoul, Korea. Environmental Research Letters 6(3). https://doi.org/doi:10.1088/17489326/6/3/034027.

Wang, C., Z. Zhang, M. Zhou, L. Zhang, P. Yin, W. Ye, and Y. Chen. 2017. Nonlinear relationship between extreme temperature and mortality in different temperature zones: A systematic study of 122 communities across the mainland of China. Science of the Total Environment 586: 96-106.

World Health Organization. 2011. International statistical classification of diseases and health related problems, 10th revision, version for 2007. http://apps.who.int/classifications/apps/icd/ icd10online2007/. Accessed 31 Oct 2017.

Wu, W., Y. Xiao, G. Li, W. Zeng, H. Lin, S. Rutherford, Y. Xu, Y. Luo, et al. 2013. Temperature-mortality relationship in four subtropical Chinese cities: A time-series study using a distributed lag non-linear model. Science of the Total Environment 449: 355-362.

Yang, J., C.Q. Ou, D. Yan, Y.X. Zhou, and P.Y. Chen. 2012. Daily temperature and mortality: A study of distributed lag non-linear effect and effect modification in Guangzhou. Environmental Health 11(1). https://doi.org/doi:10.1186/1476-069X-11-63.

Yang, J., P. Yin, M. Zhou, C.Q. Ou, M. Li, Y. Liu, J. Gao, B. Chen, et al. 2016. The effect of ambient temperature on diabetes mortality in China: A multi-city time series study. Science of the Total Environment 543: 75-82.

Yuan, W., X. Bing, Z. Chen, J. Xia, W. Xu, C. Yang, X. Wu, and F. Yang. 2014. Validation of China-wide interpolated daily climate variables from 1960 to 2011. Theoretical and Applied Climatology 119(3-4): 689-700.

Zhang, Y. 2009. An overview of the 3C-STAR project. Paper presented at the EGU General Assembly Conference, 19-24 April 2009, Vienna, Austria. 